\title{
Comparison of Localized High Volume Tumor and Locally Advanced Low Volume Tumor after Radical Prostatectomy according to Risk Classification
}

\author{
Tae Jin Kim, In Jae Lee, Byeong Do Song, Sang Chul Lee, \\ Sung Kyu Hong, Seok Soo Byun, Sang Eun Lee, Jong Jin Oh \\ Department of Urology, Seoul National University Bundang Hospital, Seongnam, Korea
}

Purpose: To investigate the percentage of pathologic tumor volume (pTPV) among patients who underwent radical prostatectomy (RP).

Materials and Methods: We reviewed 3,080 patients who underwent RP between September 2003 and March 2015 and with a postoperative follow-up for more than 1 year. The patient population was stratified into 4 disease risk groups according to tumor stage and pTPV (T2 low volume [T2LV], T2 high volume [T2HV], T3 low volume [T3LV], and T3 high volume [T3HV]). Probability of biochemical recurrence (BCR)-free survival was determined using Kaplan-Meier curves. pTPV was evaluated by Multivariate Cox proportional hazard analysis for predicting BCR. Subgroup analyses were performed according to preoperative risk.

Results: The median prostate-specific antigen (PSA) was $7.87 \mathrm{ng} / \mathrm{mL}$, and pTPV was $10 \%$. Among a total of 2,964 patients, T2LV had 1,473 (49.7\%), T2HV was $598(20.2 \%)$, T3LV with $199(6.7 \%)$, and T3HV was 694 (23.4\%). When comparing T2HV and T3LV, Gleason score and positive surgical margin rate was higher in T3LV. During a 50-month follow-up, BCR-free survival rate was higher in the T2HV group $(p<0.001)$. pTPV was a significant factor to predict BCR in multivariate Cox analysis. In subgroup analyses, T2HV group had similar BCR-free survival rates to T3LV group in the preoperative high risk group while PTPV was significant in the high risk group.

Conclusions: PTPV was a significant predictor of $B C R$ among prostate cancer patients after RP, however T2HV had favorable BCR results. Among patients with a preoperative high PSA and Gleason score, T2HV had similar BCR results to T3LV. (Korean J Urol Oncol 2016;14:165-171)

Key Words: Prostate $\cdot$ Prostate cancer $\cdot$ Recurrence $\cdot$ Tumor volume

Received October 28, 2016, Revised November 15, 2016,

Accepted December 7, 2016

Corresponding Author: Jong Jin Oh

Department of Urology, Seoul National University Bundang Hospital, 82 Gumi-ro 173beon-gil, Bundang-gu, Seongnam 13620, Korea

E-mail: urojj@snubh.org

Tel: +82-31-787-7351, Fax: +82-31-787-4057

- This work was supported by grant no 02-2016-015 from the SNUBH (Seoul National University Bundang Hospital) Research Fund and supported by the Basic Science Research Program through the National Research Foundation of Korea (NRF), funded by the Ministry of Education, Science and Technology (NRF-2014R1A1A2059658).

\begin{abstract}
INTRODUCTION
Prostate cancer ( $\mathrm{PCa})$ is the second most frequently diagnosed cancer and the fifth leading cause of cancer death in males worldwide. It was accountable for $15 \%$ of the total new cancer cases and $7 \%$ of total male cancer deaths in $2012 .{ }^{1}$ Currently, radical prostatectomy (RP) is the only surgical option for patients with localized PCa. ${ }^{2}$ While most localized PCa patients have an excellent 10-year biochemical recurrence (BCR)-free survival rates of $73 \%$ to $99 \%$, some cancers will
\end{abstract}

This is an Open Access article distributed under the terms of the Creative Commons Attribution Non-Commercial License (http://creativecommons.org/licenses/by-nc/4.0/) which permits unrestricted non-commercial use, distribution, and reproduction in any medium, provided the original work is properly cited. 2016 (C) Copyright The Korean Urological Oncology Society and The Korean Prostate Society. All Rights Reserved. 
eventually recur. ${ }^{3,4}$ Previous studies have identified variables such as prostate-specific antigen (PSA), pathological substage, Gleason score and/or positive surgical margins (PSMs) as predictors of BCR. However, the aforementioned studies had limited accuracy and predictive power. Therefore, studies concerning increased accuracy and prediction of BCR are necessary.

Traditionally, in solid organ cancer, tumor size or tumor volumes (TVs) are important factors to predict cancer related outcomes and prognosis. ${ }^{5}$ The size of a tumor is an important reflection of its biology, determined by complex interacting factors such as cell proliferation, apoptosis and angiogenesis. ${ }^{6,7} \mathrm{TV}$ at the time of treatment for clinically localized PCa could reflect important properties that influence its clinical behavior. Despite the biological relevance of tumor size, the status of TV in PCa as an independent predictor of outcome after RP has not been well defined. Some studies demonstrated a positive relationship between TV and cancer progression; ${ }^{8}$ others argued that $\mathrm{TV}$ is not an independent predictor of $\mathrm{PCa}$ progression when incorporating the more readily determined tumor grade and stage in the analysis. ${ }^{9-11}$

Additionally, several studies have reported the predictive value of the percentage of pathologic tumor volume (pTPV) in prediction of BCR. ${ }^{12-17}$ Even though TV shows potential to be a significant factor, there were no studies done on the comparison of localized high volume PCa and low volume advanced $\mathrm{PCa}$. Therefore we investigated the aforementioned issue among PCa patients who underwent RP using pTPV.

\section{MATERIALS AND METHODS}

\section{Patients}

After obtaining Institutional Review Board approval (IRB No. SNUBH-B-1612-375-103), we reviewed the data of 3,080 patients who underwent RP for PCa between September 2003 and March 2015 at Seoul National University Bundang Hospital and were postoperatively followed up for more than 1 year. The patient population was stratified into 4 disease risk groups $-\mathrm{T} 2$ low volume group (T2LV), T2 high volume group (T2HV), T3 low volume group (T3LV) and T3 high volume group (T3HV) -according to tumor stage and the median pTPV value of $10 \%$. After excluding patients who underwent neoadjuvant, adjuvant hormone or radiation therapy and pathologic T4 and node positive disease and those with inadequate data, a total of 2,964 patients were included in the final analysis.

\section{Pathologic Evaluation}

Specimens from RPs were weighed, fixed intact in $20 \%$ neural buffered formalin, and sectioned at 3- to 4-mm intervals with the apical and bladder neck portion sectioned radially to allow evaluation of margin status parallel to urethra. For each pathologic slide, the percent of tumor involvement per slide was estimated, and pTPV was determined by averaging the estimates from all slides using methods previously reported by others. $^{13,18}$

\section{Statistical Analysis}

Data assessment included patient age, preoperative PSA level, prostate specimen weight, pTPV, pathologic Gleason score, pathologic stage, surgical margin status, lymph node involvement, and postoperative follow-up PSA data, and was compared according to groups via tumor stage and pTPV by the Mann-Whitney U or Kruskal-Wallis test. BCR was defined as two consecutive rises in PSA of $0.2 \mathrm{ng} / \mathrm{mL}$ or higher at a minimum of 2 months following RP. ${ }^{19}$ Probability of BCR-free survival was determined using Kaplan-Meier curves with log rank test used to assess statistical significance. Effects of various variables on BCR-free survival were assessed using the Cox proportional hazards model. An additional subgroup analysis was performed via clinical risk classification. The SPSS ver. 15.0 (SPSS Inc., Chicago, IL, USA) was used for statistical analysis. A two-tailed $\mathrm{p}<0.05$ was considered significant for all analyses.

\section{RESULTS}

\section{Baseline Characteristics}

Patient characteristics are shown in Table 1. When stratified into groups (T2LV, T2HV, T3LV and T3HV), 1,473 patients (49.7\%) had pathologic T2 and pTPV less than 10\% (T2LV), 598 patients $(20.2 \%)$ had pathologic T2 and pTPV $\geq 10 \%$ (T2HV), 199 patients (6.7\%) were T3LV and 694 patients (23.4\%) were T3HV. Among total patients, the median PSA was $7.87 \mathrm{ng} / \mathrm{mL}$, and median pTPV was $10 \%$ (mean value; $16.9 \%)$. In pT2 patients $(\mathrm{n}=2,071)$, the T2HV group had a higher pathologic Gleason score, PSM rate and BCR rate than T2LV (p<0.001). In pT3 patients $(n=893)$, the T3HV group also had a higher pathologic Gleason score, PSM rate, seminal vesicle invasion $(\mathrm{SVI})$ and $\mathrm{BCR}$ rate than $\mathrm{T} 2 \mathrm{LV}(\mathrm{p}<0.001)$. 
Table 1. Baseline characteristics of entire patients according to tumor volume and pathologic stage

\begin{tabular}{|c|c|c|c|c|c|}
\hline Characteristic & $\mathrm{T} 2 \mathrm{LV}$ & $\mathrm{T} 2 \mathrm{HV}$ & $\mathrm{T} 3 \mathrm{LV}$ & T3HV & p-value \\
\hline No. of patients & 1,473 & 598 & 199 & 694 & \\
\hline Age $(y)$ & $65.45 \pm 6.99$ & $65.58 \pm 6.91$ & $67.36 \pm 6.08$ & $66.66 \pm 6.51$ & $<0.001$ \\
\hline Body mass index $\left(\mathrm{kg} / \mathrm{m}^{2}\right)$ & $24.43 \pm 6.85$ & $24.32 \pm 2.91$ & $25.75 \pm 16.59$ & $24.45 \pm 2.84$ & 0.059 \\
\hline Prostate specific antigen $(\mathrm{ng} / \mathrm{mL})$ & $6.21 \pm 8.84$ & $8.57 \pm 11.21$ & $7.45 \pm 6.40$ & $14.61 \pm 22.20$ & $<0.001$ \\
\hline Pathological Gleason score & & & & & $<0.001$ \\
\hline 6 & $291(19.8)$ & $28(4.7)$ & $4(2.0)$ & $6(0.9)$ & \\
\hline 7 & $1,123(76.2)$ & $524(87.6)$ & $162(81.4)$ & $474(68.3)$ & \\
\hline$\geq 8$ & $59(4.0)$ & $46(7.7)$ & $33(16.6)$ & $214(30.8)$ & \\
\hline Prostate volume $(\mathrm{mL})$ & $42.46 \pm 16.11$ & $36.19 \pm 11.46$ & $40.13 \pm 12.58$ & $38.96 \pm 13.30$ & $<0.001$ \\
\hline Tumor volume ratio (\%) & $4.88 \pm 2.85$ & $22.99 \pm 61.73$ & $7.24 \pm 2.30$ & $38.66 \pm 89.87$ & $<0.001$ \\
\hline Seminal vesicle invasion & $0(0)$ & $0(0)$ & $29(14.6)$ & $221(31.8)$ & $<0.001$ \\
\hline Positive surgical margin & $141(9.6)$ & $151(25.3)$ & $73(36.7)$ & $409(58.9)$ & $<0.001$ \\
\hline Biochemical recurrence & $87(5.9)$ & 77 (12.9) & $49(24.6)$ & $310(44.7)$ & $<0.001$ \\
\hline Mean follow-up duration (mo) & 46.25 & 46.94 & 50.11 & 45.59 & 0.480 \\
\hline
\end{tabular}

Values are presented as mean \pm standard deviation or number (\%) unless otherwise indicated.

$\mathrm{LV}$ : low volume, HV: high volume.

Table 2. Multivariate Cox proportional hazard analysis to predict biochemical recurrence after radical prostatectomy among total patients

\begin{tabular}{lcrrr}
\hline \multicolumn{1}{c}{ Variable } & Hazard ratio & 95\% CI & p-value \\
\hline Age & 0.995 & $0.981-1.008$ & 0.439 \\
Prostate-specific antigen & 1.010 & $1.006-1.013$ & $<0.001$ \\
Prostate volume & 1.002 & $0.995-1.008$ & 0.602 \\
Pathologic Gleason score & 3.409 & $1.935-6.007$ & $<0.001$ \\
Extracapsular extension & 2.553 & $2.039-3.196$ & $<0.001$ \\
Seminal vesicle invasion & 2.727 & $2.195-3.389$ & $<0.001$ \\
Positive surgical margin & 1.951 & $1.597-2.384$ & $<0.001$ \\
Positive tumor volume (10\%) & 1.569 & $1.248-1.973$ & $<0.001$ \\
\hline
\end{tabular}

CI: confidence interval.

When comparing T2HV and T3LV groups, pathologic Gleason score $(p<0.001)$, PSM $(p=0.002)$, and BCR $(p<0.001)$ were higher in the T3LV group.

\section{BCR-free Survival Analysis}

Univariate analysis showed that pTPV was associated with BCR-free survival among total patients (log rank $\mathrm{p}<0.001)$. A multivariate Cox proportional hazards regression model demonstrated that pTPV was an independent predictor of BCR-free survival $(\mathrm{p}<0.001)$ in the study population after adjusting for age, PSA, prostate volume, pathologic Gleason score, extracapsular extension, SVI, and PSM (Table 2). Among the 4 groups, BCR-free survival rates were significantly different as shown in Fig. 1 ( $\log$ rank test, all $\mathrm{p}<0.001)$. The 10 -year

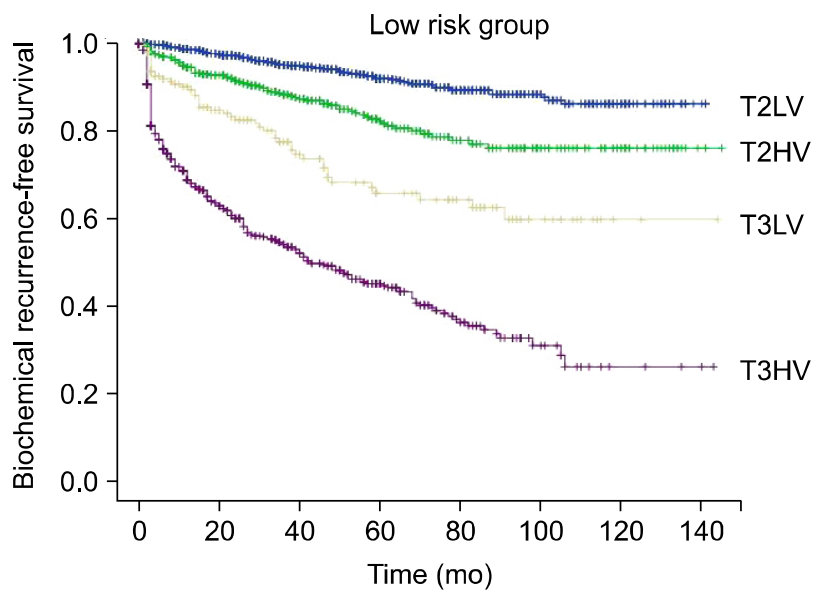

Fig. 1. Biochemical recurrence-free survival rate according to tumor stage and percentage of pathologic tumor volume. T2LV: T2 low volume group, T2HV: T2 high volume group, T3LV: T3 low volume group, T3HV: T3 high volume group.

BCR-free survival was calculated as $86.1 \%$ in T2LV, $76.0 \%$ in $\mathrm{T} 2 \mathrm{HV}, 59.7 \%$ in $\mathrm{T} 3 \mathrm{LV}$, and $26.2 \%$ in $\mathrm{T} 3 \mathrm{HV}$.

\section{Subgroup Analysis according to Risk Group}

In a subgroup analysis according to stratification of risk classification, pTPV was a significant predictor to BCR only in the high risk group and not in the low-intermediate risk group (Table 3). As shown in Fig. 2, T2LV PCa had a significantly higher BCR-free survival rate compared to other groups (vs. T2HV, p=0.003; vs. T3LV, $\mathrm{p}<0.001 ; \mathrm{T} 3 \mathrm{HV}, \mathrm{p}<0.001)$. However, in the low risk classification groups, there were no 
Table 3. Multivariate Cox proportional hazard analysis to predict biochemical recurrence after radical prostatectomy according to risk group

\begin{tabular}{|c|c|c|c|c|c|c|c|c|c|}
\hline \multirow{2}{*}{ Variable } & \multicolumn{3}{|c|}{ Low risk group } & \multicolumn{3}{|c|}{ Intermediate risk group } & \multicolumn{3}{|c|}{ High risk group } \\
\hline & HR & $95 \% \mathrm{CI}$ & p-value & HR & $95 \% \mathrm{CI}$ & $\mathrm{p}$-value & HR & $95 \% \mathrm{CI}$ & p-value \\
\hline Age & 0.964 & $0.924-1.006$ & 0.091 & 0.984 & $0.959-1.010$ & 0.236 & 1.000 & $0.983-1.018$ & 0.992 \\
\hline PSA & 1.111 & $0.961-1.284$ & 0.206 & 1.045 & $1.006^{-1.086}$ & 0.024 & 1.004 & $0.996-1.008$ & 0.087 \\
\hline Prostate volume & 0.982 & $0.955-1.010$ & 0.206 & 0.988 & $0.974-1.003$ & 0.111 & 1.004 & $0.996-1.012$ & 0.302 \\
\hline Pathologic Gleason score & 1.226 & $0.585-2.567$ & 0.590 & 3.586 & $1.983-6.484$ & $<0.001$ & 2.110 & $1.672-2.663$ & $<0.001$ \\
\hline $\mathrm{ECE}$ & 2.567 & $1.329-4.959$ & 0.005 & 2.324 & $1.601-3.375$ & $<0.001$ & 2.016 & $1.470-2.766$ & $<0.001$ \\
\hline SVI & 14.917 & $3.355-66.330$ & $<0.001$ & 1.761 & $1.058-2.932$ & 0.029 & 2.011 & $1.566-2.582$ & $<0.001$ \\
\hline PSM & 3.795 & $1.965^{-7.332}$ & $<0.001$ & 2.073 & $1.460-2.944$ & $<0.001$ & 1.547 & $1.191-2.010$ & 0.001 \\
\hline PTV (10\%) & 1.063 & $0.550-2.054$ & 0.856 & 0.828 & $0.569-1.203$ & 0.321 & 1.914 & $1.325-2.765$ & 0.001 \\
\hline
\end{tabular}

HR: hazard ratio, CI: confidence interval, PSA: prostate-specific antigen, ECE: extracapsular extension, SVI: seminal vesicle invasion, PSM: positive surgical margin, PTV: pathologic tumor volume.
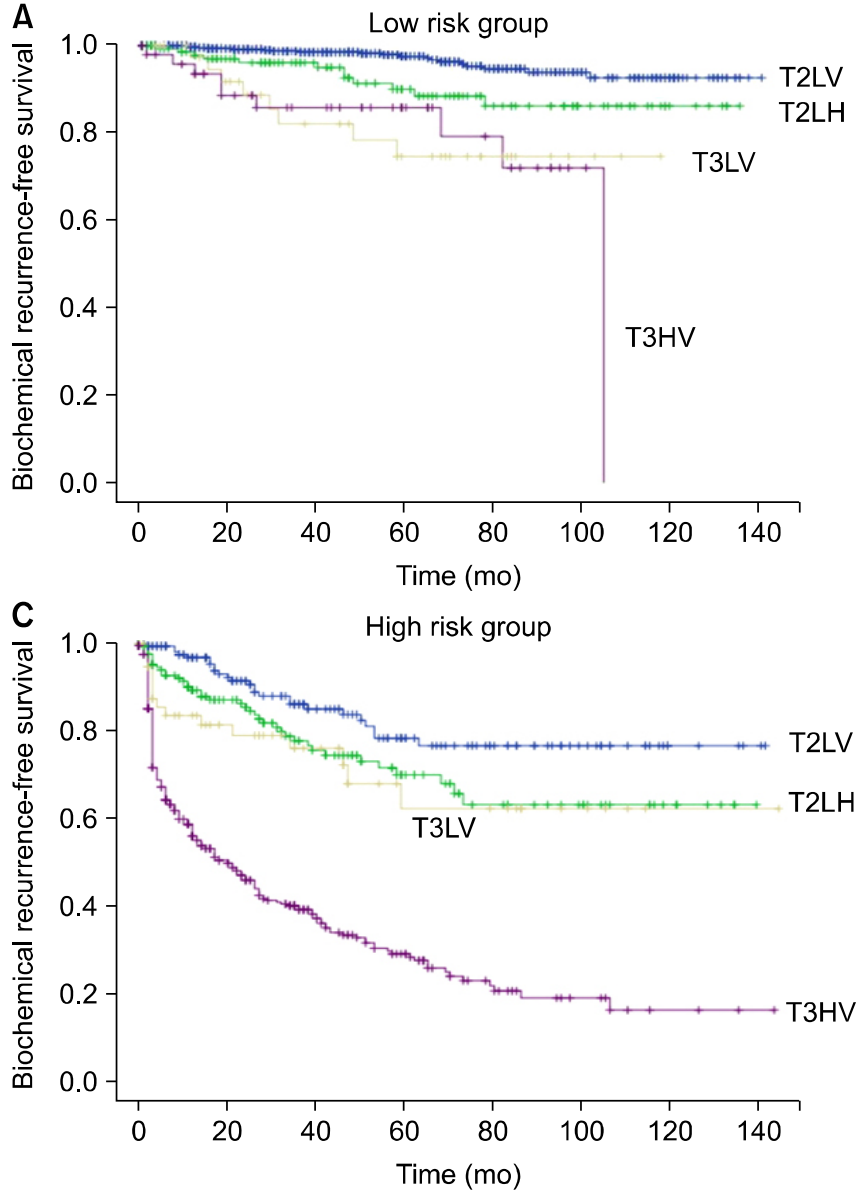

significant differences. In the intermediate risk group, T2LV and T2HV had similar BCR-free survival rate and T3LV and T3HV had also similar results for BCR rate. Cox proportional multivariate analysis also showed that PTPV was not a significant predictor to BCR in the low and intermediate risk group (Table 3). However in the high risk group, the T2HV

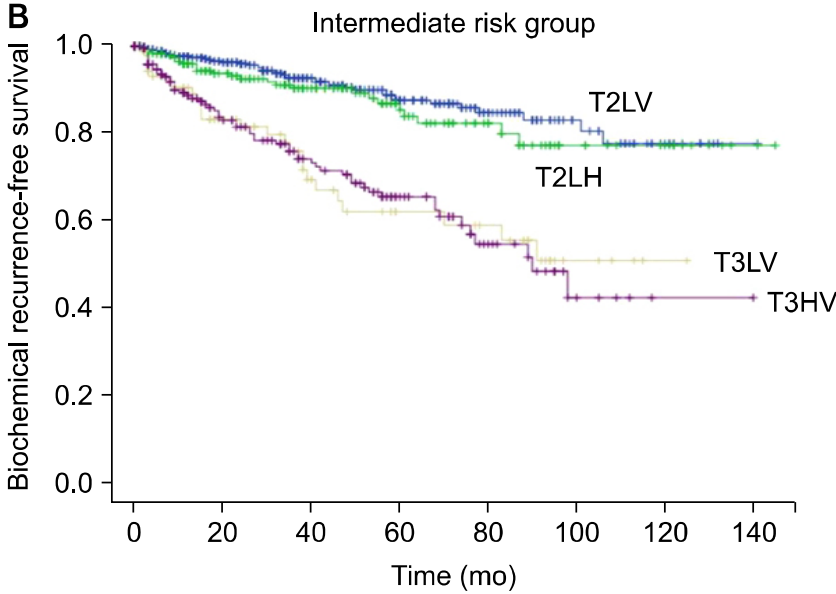

Fig. 2. Biochemical recurrence-free survival rate according to tumor stage and percentage of pathologic tumor volume. T2LV: $\mathrm{T} 2$ low volume group, T2HV: T2 high volume group, T3LV: T3 low volume group, T3HV: T3 high volume group.

group had similar BCR-free survival rates when compared to the T3LV group (Fig. 2C) $(\mathrm{p}=0.543)$. As shown in Table 3, pTPV was a significant predictor to BCR only in the high risk group (hazard ratio, 1.914; 95\% confidence interval, 1.325$2.765 ; \mathrm{p}<0.001)$ after adjusting for other factors. 


\section{DISCUSSION}

In our study, pTPV was observed to be an independent predictor of BCR-free survival following RP among the total subjects. In high pTPV organ confined PCa, BCR free survival rates are favorable compared to locally advanced PCa regardless of pTPV. However in high risk groups who have a higher PSA and Gleason score, PTV could be a significant factor to predict BCR, and our study shows that T2HV disease had similar BCR related results compared to T3LV after RP.

Our finding that TV is an independent prognosticator of BCR agrees with several independent studies. ${ }^{8,20-22}$ Humphrey et al. $^{23,24}$ reported that intraglandular tumor extent measured as percent of carcinoma correlated with disease progression following RP among patients with clinically localized PCa. Moreover, Carvalhal et al. ${ }^{25}$ and Ramos et al. ${ }^{13}$ observed that pTPV was an independent predictor of BCR among patients who underwent RP for pathologically organ confined disease. Furthermore, a study done by Rampersaud et al. ${ }^{18}$ showed that pTPV was a significant factor in disease progression following RP even after adjusting for multiple clinical and pathological characteristics for margin positive or extraprostatic extension of tumor as well as those with pathologically organ-confined disease. On the other hand, Epstein et al. ${ }^{9}$ reported from their series of clinically localized PCa patients treated with RP that PTV did not provide independent information beyond that offered by other factors.

The aforementioned studies are a reflection of the ongoing controversy surrounding the actual value of pTPV as a potential prognostic indicator in patients who underwent RP. There are several possible explanations for the disagreement of the role of volume in risk of recurrence. One explanation of these differences is in the methods used to measure TV. In several series, objective measures of TV, such as by computerized planimetry or measurement of the greatest tumor dimension, have demonstrated prediction of BCR after RP independent of other clinical and pathological variables. ${ }^{26,27}$ Subjective measures, such as visual estimates of tumor size or percentage of gland involvement, have failed to provide prognostic information, although the literature is conflicting. ${ }^{25}$ A second explanation could be due to a small study cohort or small $\mathrm{TV}$ in series in which volume has failed to correlate with outcome. Finally, some studies include large numbers of patients with relatively low grade cancers. Merrill et al. ${ }^{15}$ found that in patients with a pathologic Gleason score of 6 or less, TV failed to predict for BCR. Therefore, the inclusion of patients with Gleason grade $3+3$ disease in prognostic models will corrupt the predictive power of TV.

Results from our study show that pTPV was an independent predictor of BCR-free survival following RP. However, the predictive value of pTPV was only significant in the high risk D'Amico classified group, which shows that pTPV could be a significant factor to predict BCR in highly aggressive tumors and in the low or intermediate risk groups, tumor staging is a much more important factor compared to TV.

The Gleason grade represents tumor differentiation while pTPV reflects the tumor burden; these are principal outcome determinants in any solid organ cancer. Pathologic stage represents the disease extent resulting directly from the combination of both factors; all other clinicopathologic factors may have potential to influence outcome. Therefore, in pT2 $\mathrm{PCa}$, prognosis may be primarily determined by pTPV and the Gleason grade; T2HV disease with high a PSA and Gleason score disease should treated and managed in similar fashion to locally advanced PCa.

TV or size is considered as an important prognostic indicator in many cancer types and is often included in pathological staging. ${ }^{20-22}$ However, the importance of $\mathrm{TV}$ in the prognosis of PCa has been debated. The main reason for this ongoing debate has been a lack of consensus on the best way to assess cancer volume or extent. ${ }^{28}$ Some proposed the direct measurements of TV, while others have used percent cancer as a ratio of TV to total prostate volume as an estimate of the percentage of the gland involved with cancer. ${ }^{21,22}$ To date, TV and percent cancer have not been compared directly.

Potential limitations of our study include the retrospective nature of study design. Moreover, we could not control for intra- or interobserver variability in assessing pTPV. Although pTPV in all specimens were calculated by an experienced single uropathologist, pTPV measurement done by computerized morphometric methods would be regarded as a more accurate estimate of the actual tumor burden. The present study was a retrospective analysis with a short median follow-up duration was 48 months in our study. Due to the lack of long term follow up, time to BCR rather than cancer-specific survival was designated as the primary endpoint in our study. A final limitation of the present study is that the different number of sub- 
jects according to each group could affect the interpretation of our results. Therefore, the results of this study should be validated in a large scaled cohort study.

\section{CONCLUSIONS}

The present study demonstrated that pTPV was a significant predictor of BCR among patients who underwent RP. Patients with a high PTPV, organ confined disease had a favorable BCR-free survival rate compared to locally advanced PCa regardless of pTPV. However in the high risk group, T2HV disease had similar BCR related results when compared to T3LV after RP, therefore, patients with T2HV with a high PSA and Gleason score disease should be approached and managed similarly to locally advanced PCa.

\section{CONFLICT OF INTEREST}

The authors claim no conflicts of interest.

\section{REFERENCES}

1. Torre LA, Bray F, Siegel RL, Ferlay J, Lortet-Tieulent J, Jemal A. Global cancer statistics, 2012. CA Cancer J Clin 2015;65:87-108

2. Heidenreich A, Bastian PJ, Bellmunt J, Bolla M, Joniau S, van der Kwast $\mathrm{T}$, et al. EAU guidelines on prostate cancer. part 1: screening, diagnosis, and local treatment with curative intent-update 2013. Eur Urol 2014;65:124-37

3. Han M, Partin AW, Zahurak M, Piantadosi S, Epstein JI, Walsh PC. Biochemical (prostate specific antigen) recurrence probability following radical prostatectomy for clinically localized prostate cancer. J Urol 2003;169:517-23

4. Caso JR, Tsivian M, Mouraviev V, Polascik TJ, Moul JW. Pathological T2 sub-divisions as a prognostic factor in the biochemical recurrence of prostate cancer. BJU Int 2010;106: 1623-7

5. Lee KC, Kim HJ, Sung K, Choi YE, Lee SH, Lim S, et al. The predictive value of tumor size, volume, and markers during radiation therapy in patients with cervical cancer. Int $\mathbf{J}$ Gynecol Cancer 2016 Oct 18 [Epub]. http://doi.org/10.1097/ IGC. 0000000000000837

6. Nicholson B, Theodorescu D. Angiogenesis and prostate cancer tumor growth. J Cell Biochem 2004;91:125-50

7. Abdulkadir SA, Carvalhal GF, Kaleem Z, Kisiel W, Humphrey PA, Catalona WJ, et al. Tissue factor expression and angiogenesis in human prostate carcinoma. Hum Pathol 2000;31:443-7
8. Stamey TA, McNeal JE, Yemoto CM, Sigal BM, Johnstone IM. Biological determinants of cancer progression in men with prostate cancer. JAMA 1999;281:1395-400

9. Epstein JI, Carmichael M, Partin AW, Walsh PC. Is tumor volume an independent predictor of progression following radical prostatectomy? A multivariate analysis of 185 clinical stage B adenocarcinomas of the prostate with 5 years of followup. J Urol 1993;149:1478-81

10. Graefen M, Karakiewicz PI, Cagiannos I, Klein E, Kupelian PA, Quinn DI, et al. Validation study of the accuracy of a postoperative nomogram for recurrence after radical prostatectomy for localized prostate cancer. J Clin Oncol 2002;20: 951-6

11. Salomon L, Levrel O, Anastasiadis AG, Irani J, De La Taille A, Saint F, et al. Prognostic significance of tumor volume after radical prostatectomy: a multivariate analysis of pathological prognostic factors. Eur Urol 2003;43:39-44

12. Ahyai SA, Zacharias M, Isbarn H, Steuber T, Eichelberg C, Köllermann J, et al. Prognostic significance of a positive surgical margin in pathologically organ-confined prostate cancer. BJU Int 2010;106:478-83

13. Ramos CG, Roehl KA, Antenor JA, Humphrey PA, Catalona WJ. Percent carcinoma in prostatectomy specimen is associated with risk of recurrence after radical prostatectomy in patients with pathologically organ confined prostate cancer. J Urol 2004;172:137-40

14. Palisaar RJ, Graefen M, Karakiewicz PI, Hammerer PG, Huland E, Haese A, et al. Assessment of clinical and pathologic characteristics predisposing to disease recurrence following radical prostatectomy in men with pathologically organ-confined prostate cancer. Eur Urol 2002;41:155-61

15. Merrill MM, Lane BR, Reuther AM, Zhou M, Magi-Galluzzi C, Klein EA. Tumor volume does not predict for biochemical recurrence after radical prostatectomy in patients with surgical Gleason score 6 or less prostate cancer. Urology 2007;70: 294-8

16. Song C, Kang T, Yoo S, Jeong IG, Ro JY, Hong JH, et al. Tumor volume, surgical margin, and the risk of biochemical recurrence in men with organ-confined prostate cancer. Urol Oncol 2013;31:168-74

17. Chun FK, Briganti A, Jeldres C, Gallina A, Erbersdobler A, Schlomm T, et al. Tumour volume and high grade tumour volume are the best predictors of pathologic stage and biochemical recurrence after radical prostatectomy. Eur J Cancer 2007; 43:536-43

18. Rampersaud EN, Sun L, Moul JW, Madden J, Freedland SJ. Percent tumor involvement and risk of biochemical progression after radical prostatectomy. J Urol 2008;180:571-6

19. Cookson MS, Aus G, Burnett AL, Canby-Hagino ED, D'Amico AV, Dmochowski RR, et al. Variation in the definition of biochemical recurrence in patients treated for localized prostate cancer: the American Urological Association 
Prostate Guidelines for Localized Prostate Cancer Update Panel report and recommendations for a standard in the reporting of surgical outcomes. J Urol 2007;177:540-5

20. D'Amico AV, Whittington R, Malkowicz SB, Schultz D, Kaplan I, Beard CJ, et al. Calculated prostate cancer volume greater than $4.0 \mathrm{~cm} 3$ identifies patients with localized prostate cancer who have a poor prognosis following radical prostatectomy or external-beam radiation therapy. J Clin Oncol 1998;16:3094-100

21. Ates M, Teber D, Gözen AS, Tefekli A, Sugiono M, Hruza $\mathrm{M}$, et al. Do tumor volume, tumor volume ratio, type of nerve sparing and surgical experience affect prostate specific antigen recurrence after laparoscopic radical prostatectomy? A matched pair analysis. J Urol 2007;177:1771-5

22. Guzzo TJ, Vira MA, Neway W, Hwang WT, Tomaszewski $\mathrm{J}$, VanArsdalen $\mathrm{K}$, et al. Minimal tumor volume may provide additional prognostic information in good performance patients after radical prostatectomy. Urology 2007;69:1147-51

23. Humphrey PA, Vollmer RT. Intraglandular tumor extent and prognosis in prostatic carcinoma: application of a grid method to prostatectomy specimens. Hum Pathol 1990;21:799-804

24. Humphrey PA, Walther PJ, Currin SM, Vollmer RT. Histologic grade, DNA ploidy, and intraglandular tumor extent as indicators of tumor progression of clinical stage B prostatic carcinoma. A direct comparison. Am J Surg Pathol 1991;15:1165-70

25. Carvalhal GF, Humphrey PA, Thorson P, Yan Y, Ramos CG, Catalona WJ. Visual estimate of the percentage of carcinoma is an independent predictor of prostate carcinoma recurrence after radical prostatectomy. Cancer 2000;89:1308-14

26. Noguchi M, Stamey TA, McNeal JE, Yemoto CE. Assessment of morphometric measurements of prostate carcinoma volume. Cancer 2000;89:1056-64

27. Renshaw AA, Richie JP, Loughlin KR, Jiroutek M, Chung A, D'Amico AV. The greatest dimension of prostate carcinoma is a simple, inexpensive predictor of prostate specific antigen failure in radical prostatectomy specimens. Cancer 1998;83:748-52

28. Grignon DJ, Sakr WA. Pathologic staging of prostate carcinoma. What are the issues? Cancer 1996;78:337-40 